\section{Branch retinal vein occlusion: the importance of the topographical distribution of retinal vessels among risk factors}

${ }^{1}$ Faculty of Medicine,

Department of Ophthalmology, Ege University, Izmir, Turkey

${ }^{2}$ Hakkari Government Hospital, Hakkari, Turkey

Correspondence:

Z Oztas, Ege Universitesi Tip Fakultesi, Goz Hast AD, 35040 Bornova, Izmir Turkey

Tel: +90 55320329 00;

Fax: +90 2323881469 .

E-mail: zaferdr2000@gmail. com

Received: 4 June 2016 Accepted in revised form: 7 November 2016;

Published online:

13 January 2017

A preliminary results of this study was presented at the 15th EURETINA CongressNice 2015.

\begin{abstract}
Purpose To investigate the role of the topographical distribution of temporal retinal vessels in anatomical predisposition to branch retinal vein occlusion (BRVO).

Patients and methods Sixty patients with BRVO and 60 control subjects were included in this retrospective-observational study. The fundus images of the individuals were classified into four topographical vessel positions: P1, the superior and inferior temporal retinal veins were closer to the foveal center; $\mathbf{P 2}$, the superior and inferior temporal retinal arteries were closer to the foveal center; and P3 and P4, the superior temporal retinal vein and inferior temporal retinal artery were closer to the foveal center or vice versa. The groups were compared in terms of demographics and topographical vessel positions.

Results There were no significant differences between the groups in terms of age and gender $(P>0.05)$. The topographical distribution of temporal retinal vessels among the BRVO and control eyes were significant $(P<0.001)$. P1 was less common in BRVO eyes (5\%) when compared with control eyes (33.3\%); however, P2 was more common in BRVO eyes (46.7 vs $20 \%$ ). There were no significant differences in terms of the distribution of $\mathrm{P} 3$ and $\mathrm{P} 4$ vessel positions in the BRVO and control groups $(P>0.05)$. A logistic regression test revealed that the risk of BRVO increases 15-fold in P2, 6-fold in P3, and 8-fold in P4 when compared with eyes having P1.

Conclusion P1 eyes are less likely to be affected by BRVO. Therefore, the topographical distribution of retinal vessels can be assessed as a risk factor for BRVO.
\end{abstract}

Z Oztas', C Akkin'1, S Nalcaci', O llim² and $\mathrm{F}$ Afrashi ${ }^{1}$

Eye (2017) 31, 726-731; doi:10.1038/eye.2016.318; published online 13 January 2017

\section{Introduction}

Retinal vein occlusion is the second most common cause of visual loss classified under retinal vascular disorders after diabetic retinopathy. ${ }^{1,2}$ Retinal vein occlusion may be divided into three types on the basis of the site of occlusion: (1) branch retinal vein occlusion (BRVO); (2) central retinal vein occlusion (CRVO); and (3) hemi-CRVO, which is considered as a subgroup of $\mathrm{CRVO} .^{3,4}$

The five-year incidence of any retinal vein occlusion is $\sim 1 \%$, as determined by populationbased studies. 5,6 The incidence of BRVO is generally higher than the incidence of CRVO. ${ }^{1}$ The prevalence of retinal vein occlusion ranges from 0.3 to $2.1 \%{ }^{7,8}$ in populations older than 40 , and the incidence of BRVO is 3-10 times ${ }^{9,10}$ higher. Overall, the prevalence as well as the incidence of retinal vein occlusion increases with age. ${ }^{5,11,12}$

$\mathrm{BRVO}$, grouped as major or macular types, is a relatively common form of retinal vein occlusion. Many risk factors have been investigated and described as being associated with BRVO. ${ }^{4}$ The aim of this study is to investigate the importance of the topographical distribution of the main temporal retinal vessels in patients with BRVO.

\section{Methods}

After obtaining approval from the Ege University Faculty of Medicine, Research Ethics Committee, 60 patients (31 male, 29 female) with 
BRVO and 60 age-matched control subjects

(23 male, 37 female) without BRVO were included in this retrospective, observational study. The BRVO group consisted of 36 right eyes and 24 left eyes with major or macular BRVO, and 32 healthy right eyes and 28 left eyes were included in the control group. The mean ages of the BRVO and control groups were $65.5 \pm 7.6$ (range: $45-80$ ) and $67.9 \pm 6.4$ (range: $55-78$ ) years, respectively. We enrolled the patients who were diagnosed with BRVO and who were over 45 years of age. Patients with ages between 45 and 65 were included only if they were fully investigated for secondary pathologies such as thrombophilia and uveitis. Patients with hypertension and/or diabetes mellitus were included. The exclusion criteria included unclear fundus photography, bilateral BRVOs, hemi-CRVOs, and younger patients with BRVO (45 years or younger) whose disorder might have been due to the secondary abnormalities as noted above. None of the individuals had a history of glaucoma, uveitis, optic disc abnormalities such as optic disc drusen and pits, or other posterior segment pathologies such as proliferative diabetic retinopathy that could interfere with their ocular structure.

Control group patients were composed of the patients in similar age groups who had come to our clinic for eye examinations, such as presbyopia or other simple refractive errors. The control group consisted of one eye from individuals who had no findings of BRVO in both of their eyes or other eye diseases that could interfere with the eye structure. The laterality of the control eyes (right eye or left eye) were randomized based on the quality of the fundus images.

All of the patients and controls were screened using digital fundus photography (Topcon 3D OCT-2000,
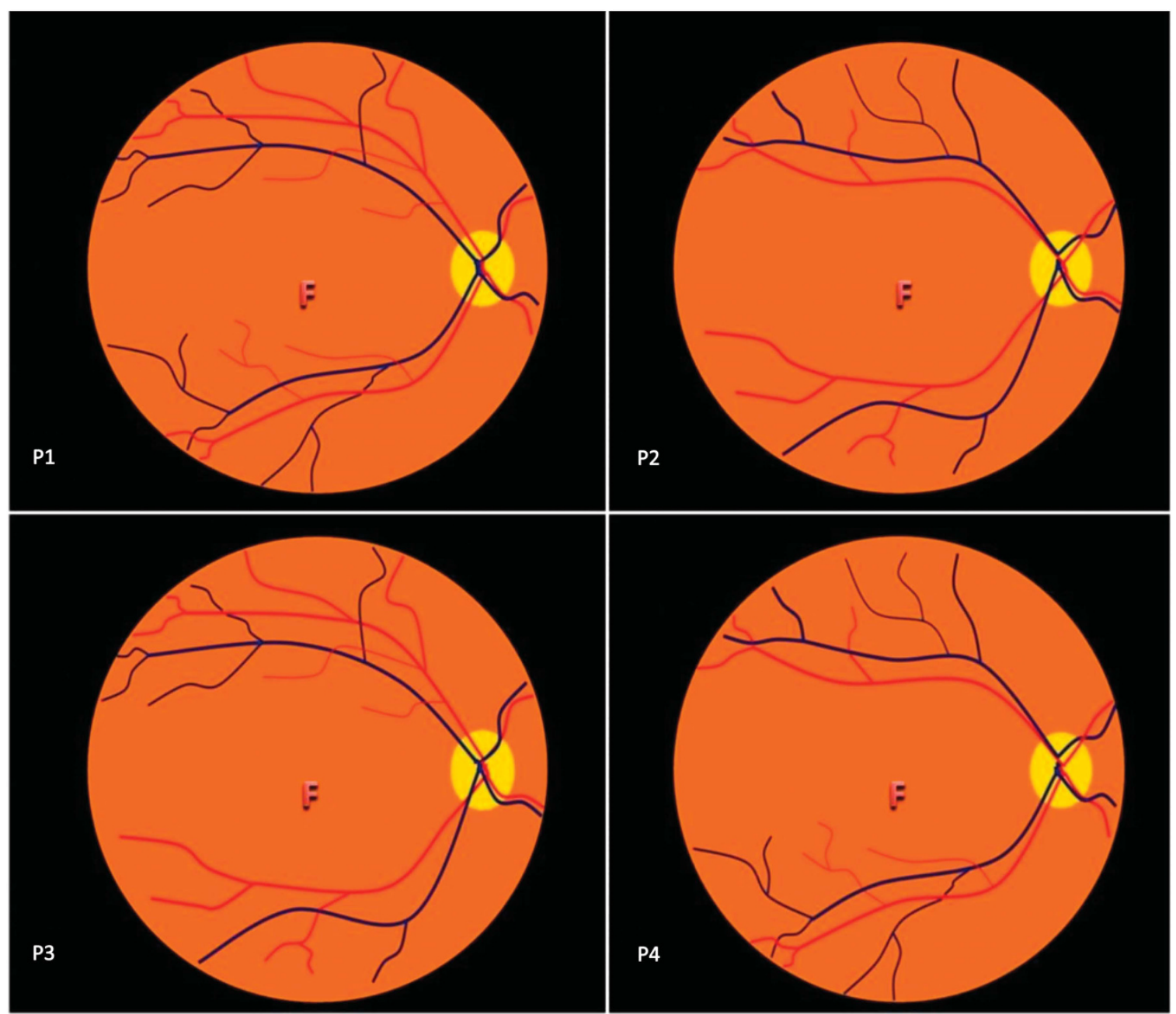

Figure 1 The four topographical vessel positions: P1, the superior and inferior temporal retinal veins were inside relative to the foveal center; P2, the superior and inferior temporal retinal arteries were inside relative to the foveal center; P3, the superior temporal retinal vein and inferior temporal retinal artery were closer relative to the foveal center; and P4, the superior temporal retinal artery and inferior temporal retinal vein were closer relative to the foveal center. 
Tokyo, Japan) between January 2010 and 2014. Forty-five degree fovea-centered fundus images of each eye were obtained. All fundus images clearly showed the optic nerve head, superior and inferior temporal retinal vessels, and the entire macular region.

In this pilot study, we grouped the retinal images into four topographical retinal vessel positions (P1, P2, P3, and $\mathrm{P} 4)$ according to the outer or inner course of the main temporal retinal arteries and veins relative to the foveal center: (P1) the superior and inferior temporal retinal veins were inside relative to the foveal center; (P2) the superior and inferior temporal retinal arteries were inside relative to the foveal center; (P3) the superior temporal retinal vein and inferior temporal retinal artery were closer to the foveal center; and (P4) the superior temporal retinal artery and inferior temporal retinal veins were closer to the foveal center (Figure 1). The assessment of the topographical retinal vessel differences in the fundus images of the both groups was performed by two experienced ophthalmologists ( $\mathrm{ZO}$ and $\mathrm{CA}$ ). The eyes were included only if the two physicians agreed with the same anatomical category.

Further evaluation of BRVO patients and the controls was required to explore a possible association between these topographic position types and arteriovenous $(\mathrm{a} / \mathrm{v})$ crossing points according to our primary results. A/v crossings can be classified on the basis of artery position into two types: the artery lies anterior to a vein (toward the vitreous cavity) and the vein lies anterior to an artery. We measured the number of these two types of a/v crossings. However, the data of first type (ie, 'the artery lies anterior to a vein') was used in statistical analyses due to low incidence of the second type mentioned above.

We performed statistical analysis using SPSS software version 16.0 (SPSS, Inc., Chicago, Illinois, USA). All of the data were reported as average $\pm \mathrm{SD}$. The mean ages of the groups were compared with $t$-test. We used $\chi^{2}$-tests to assess the gender, laterality of the eyes, and anatomical differences of topographic retinal vessel positions between each group. We also used a binary logistic regression test to detect risk of BRVO in different retinal vessel positions. The difference in mean number of $\mathrm{a} / \mathrm{v}$ crossing sites between the groups was analyzed with a one-way analysis of variance (ANOVA) test. Another binary logistic regression test was conducted to determine the effect retinal vessel positions and the number of a/v crossings as a risk of BRVO. Finally, the eyes were classified into two groups according to the number of a/v crossing sites, that is, greater than or less than 4 . A $P$-value less than 0.05 was considered to be statistically significant for all tests.

\section{Results}

There were no significant differences between the groups in terms of demographic data, including age, gender, and laterality of the eyes $(P=0.06, P=0.142$, and $P=0.461$, respectively). BRVO patients had major BRVO in $42(70 \%)$ eyes and macular BRVO in $18(30 \%)$ eyes. In the BRVO group, the occlusion was in the superior quadrant in 43 $(71.7 \%)$ eyes, while it was in the inferior quadrant in 17 (28.3\%) eyes.

The differences in the topographical distribution of temporal retinal vessels were statistically significant between the BRVO and control eyes $(P<0.001)$. As seen in Table 1, P1 was significantly less common in the BRVO eyes (5\%) when compared with the control eyes (33.3\%); however, P2 was significantly more common in BRVO eyes $(46.7 \%)$ when compared with the control eyes (20\%) (Figures 2 and 3). These results suggest that the presence of P1 has an protective effect for the occurrence of BRVO. Therefore, a binary logistic regression analysis was conducted to identify the risk of BRVO in the different retinal vessel positions. When P1 was determined as the reference, the odds ratio (OR) was 15.55 (95\% $\mathrm{Cl}=3.87-$ 62.40) for the position $\mathrm{P} 2,5.71$ (95\% Cl=1.35-24.06) for $\mathrm{P} 3$, and 8.09 (95\% $\mathrm{Cl}=1.98-32.97)$ for $\mathrm{P} 4$. There were no significant differences in terms of the distribution of the $\mathrm{P} 3$ and P4 vessel positions in the BRVO and control groups $(P=0.658$ and $P=0.532$, respectively).

In this study, all BRVO eyes (100\%) had the artery anterior to the vein at the site of the occlusion. The mean

Table 1 Distribution of the topographical retinal vessels with the number of arteriovenous crossing sites

\begin{tabular}{lccccc}
\hline $\mathrm{P}$ & \multicolumn{2}{c}{ BRVO eyes } & $\mathrm{P}$ & \multicolumn{2}{c}{ Control eyes } \\
\cline { 2 - 5 } & n. eyes $(\%)$ & n. of a/v crossings \pm SD & & n. eyes (\%) & n. of a/v crossings \pm SD \\
\hline P1 & $3(5.0 \%)^{\mathrm{a}}$ & $7.3 \pm 1.5$ & $\mathrm{P} 1$ & $20(33.3 \%)^{\mathrm{a}}$ & $2.2 \pm 1.8$ \\
P2 & $28(46.7 \%)^{\mathrm{a}}$ & $5.6 \pm 1.2$ & $\mathrm{P} 2$ & $12(20.0 \%)^{\mathrm{a}}$ & $3.2 \pm 1.4$ \\
P3 & $12(20.0 \%)$ & $5.3 \pm 1.1$ & $\mathrm{P} 3$ & $14(23.3 \%)$ & $2.9 \pm 1.8$ \\
P4 & $17(28.3 \%)$ & $5.5 \pm 2.0$ & $\mathrm{P} 4$ & $14(23.3 \%)$ & $2.5 \pm 1.5$ \\
Total & $60(100 \%)$ & $5.6 \pm 1.5^{\mathrm{b}}$ & Total & $60(100 \%)$ & $2.6 \pm 1.6^{\mathrm{b}}$ \\
\hline
\end{tabular}

Abbreviations: a/v, arteriovenous; BRVO, branch retinal vein occlusion; n, number; $\mathrm{P}$, retinal vessel position; $\mathrm{SD}$, standard deviation. ${ }^{\mathrm{a}}$ The distribution of $P$ was significantly different between the BRVO and control eyes $\left(\chi^{2}, P<0.001\right)$. ${ }^{\mathrm{b}}$ The mean number of total a/v crossings that contained a retinal artery lying anterior to the vein (toward the vitreous cavity) was $\sim 3$ units higher in the BRVO eyes when compared with the control eyes (one-way ANOVA, $P<0.001$ ). 


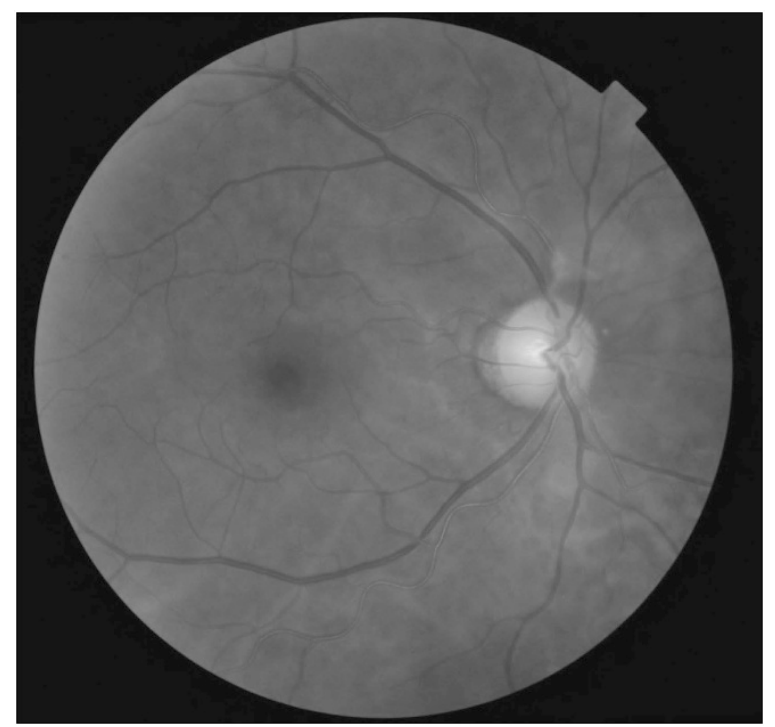

Figure 2 P1 position in which the superior and inferior temporal retinal veins were inside relative to the foveal center in the right eye of a 65-year-old man with hypertension.

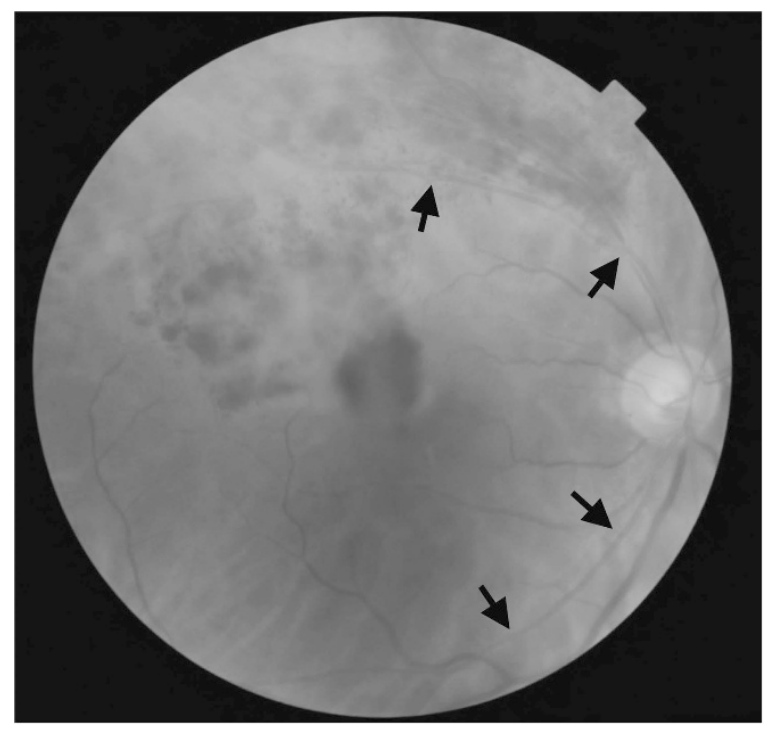

Figure 3 A 75-year-old man with superior temporal retinal vein occlusion that exhibited a P2 position in which the superior and inferior temporal retinal arteries (black arrows) were inside relative to the foveal center in his right eye.

number of total a/v crossings that contained a retinal artery lying anterior to the vein (toward the vitreous cavity) was $\sim 3$ units higher in the BRVO eyes (5.6 vs 2.6) when compared with the control eyes $(P<0.001)$. To substantiate our results, we analyzed the association between the number of a/v crossings and the retinal vessel positions in a binary logistic regression model.
The test showed that the risk of BRVO increased in eyes that had four or more $\mathrm{a} / \mathrm{v}$ crossings and contained a retinal artery lying anterior to the vein $(P<0.001$, $\mathrm{OR}=30.59,95 \% \mathrm{Cl}=8.20-114.02)$. When $\mathrm{P} 1$ was determined as the reference in this set up, the ORs were $7.37(95 \% \mathrm{Cl}=1.45-37.40)$ for $\mathrm{P} 2,3.30(95 \% \mathrm{Cl}=0.61-17.91)$ for $\mathrm{P} 3$, and 6.05 (95\% $\mathrm{Cl}=1.12-32.64)$ for $\mathrm{P} 4$.

\section{Discussion}

This study sought to assess the importance of the topographical distribution of temporal retinal vessels in anatomical risk factors of BRVO. We found that the topographical distribution of temporal retinal vessels might be an important predisposing risk factor for developing BRVO. The P1 position was found in only a few cases of BRVO (5\%), and it appears to be a protective factor for the occurrence of BRVO. The risk of BRVO increases 15-fold in P2, 6-fold in P3, and 8-fold in P4 when compared with the eyes with P1.

Furthermore, when the number of a/v crossing sites (which had an artery that lay anterior to a vein) was included in the test, the risk of BRVO increased 30 times in eyes with four or more a/v crossings, and 7-fold in P2, 3-fold in the P3, and 6-fold in P4 when compared with the eyes with P1.

A strong relationship between retinal vascular anatomical features and the BRVO has been reported in the literature. ${ }^{13-16}$ These studies were mainly focused on the a/v crossing sites and their role in the pathogenesis of BRVO. As a result of atherosclerosis, the stiffness of rigid, thick-walled arteries causes venous compression in the common adventitial sheath at the site of $\mathrm{a} / \mathrm{v}$ crossings. ${ }^{17-19}$ In almost all BRVO eyes, the artery is anterior to the vein at the site of an occlusion. ${ }^{20,21}$ Zhao et $a l^{20}$ concluded that other risk factors must play a role in the pathogenesis of BRVO because the artery lies anterior to the vein in $\sim 60 \%$ of normal a/v crossings without BRVO. As in previous studies, we observed that the artery is anterior to the vein at the site of occlusion in all eyes, and the number of these crossings was increased in the BRVO eyes.

Recently, there have been reports in the literature on the basis of optical coherence tomography (OCT) scans of a/v crossing points, proposing novel hypotheses for the pathophysiology of BRVO. 22,23 Kumagai $e t a^{22}$ reported that in healthy eyes, the retinal veins abruptly changed their directions to pass the retinal artery and frequently displayed focal luminal narrowing with no compression or flattening at the $\mathrm{a} / \mathrm{v}$ crossing area. Consistently, Muraoka et al ${ }^{23}$ demonstrated that the venous lumen was generally preserved (showing no compression), but was displaced to the outer retinal layers in eyes with BRVO of the 
artery overcrossings. In contrast to the previous studies, the authors concluded that mechanical compression of the artery may play a less important role in the pathogenesis of BRVO at the arterial overcrossing site, and hemodynamic changes downstream of the $\mathrm{a} / \mathrm{v}$ crossing may be essential in the pathogenesis of BRVO. ${ }^{23}$

This study claimed that the difference in the topographical distribution of the main temporal retinal vessels may have a role in the occurrence of BRVO. The present findings seem to be consistent with other research that found anatomical predispositions in BRVO, and the distribution of the vessels may influence the theories dependent on the venous compression or the direction of blood stream, as mentioned above. The position P1 was significantly less frequent in BRVO eyes. Only three patients demonstrated P1 in the BRVO group with an increased number of a/v crossings. However, the control eyes having P1 demonstrated a decreased number of a/v crossings. We can accordingly conclude that the presence of the P1 position with fewer number of a/v crossings might be a protective anatomical factor for BRVO.

Furthermore, P2 was more frequent in BRVO eyes, and we claimed that the eyes with P2 carried greater risk for BRVO. It seems plausible that these results might be due to the embryological organization of retinal blood vessels. These vessels spread outward from the disc and reach the peripheral retina (mostly) at birth. During this period, if the superior and inferior temporal retinal veins were closer to the foveal center (ie, the position $\mathrm{P} 1$ ), the number of a/v crossings might be fewer than in the P2 position in which the superior and inferior temporal retinal arteries were closer to the foveal center. The observed increase in the number of $\mathrm{a} / \mathrm{v}$ crossings for P2 may be attributed to the fractal properties of retinal vessels. ${ }^{24} \mathrm{~A}$ fractal can be defined as a pattern of expanding symmetry. As a result of the characteristics of a fractal, the branching patterns of retinal arteries demonstrated more a/v crossings in P2 due to the geometrical pattern of the retinal vessels. Despite all these hypotheses, some anatomical variations can be seen among the individuals.

We considered that $\mathrm{P} 3$ and $\mathrm{P} 4$ are anatomical variants of P1 and P2. The position P3 showed an appereance of $\mathrm{P} 1$ in the superior arm of the main temporal vessels and the appearance of P2 in the inferior arm of the main temporal vessels. Accordingly, the position P4 has an apperarence of $\mathrm{P} 2$ in the superior arm and an apperarence of $\mathrm{P} 1$ in the inferior arm of the main temporal vessels. Therefore, these anatomical positions 'P3 and P4' demonstrated properties of P1 or P2 in one arm of the main temporal retinal vessels. In the present study, P4 carried an increased risk of BRVO when compared with the P3 position. It is well known that most BRVOs occur in the superior and temporal part of the retina. Consistent with this, $75 \%$ of the occlusions occurred in eyes with P2 and P4 (like P2 in the superior arm) in our study. We believe that P2 is the main topographical retinal vessel position that increases the number of $\mathrm{a} / \mathrm{v}$ crossing points and also the risk of BRVO.

In fellow eyes of the BRVO group, the distribution of retinal vessel positions was similar with the control group; however, the mean number of a/v crossings was higher compared with the control group. The data were not presented in the results due to presence of potential predisposition for BRVO in fellow eyes.

With these results, a physician can simply inform their patients if they carry any risk of BRVO (eg, patients with hypertension) using a color fundus photograph. The predictive using the evaluation of the vessel position alone was found to be $75 \%$, while it grew to $90 \%$ using analyses of vessel position and the number of a/v crossing sites together. In addition, the topographical distribution of the main temporal retinal vessels can be used as a prognostic tool to determine venous occlusion risk in patients at risk of BRVO (eg, fellow eyes of the patients with BRVO).

The current investigation was limited by a relatively small sample size and its retrospective design. The main cause of the small sample size was due to the exclusion of unclear fundus images, which did not allow the evaluation of anatomical properties. To eliminate the bias factor of the retrospective design, fundus images were analyzed by two physicians. The eyes were categorized within one anatomical group when the physicians agreed. The eyes included only one anatomical group when the physicians agreed with the same category. Large-scale, wellcontrolled prospective studies are needed to confirm these findings, and additional follow-up studies are accordingly recommended to explore the associations between the topographical retinal vessel positions and other anatomical risk factors, such as a/v crossings.

In conclusion, the topographical distribution of temporal retinal vessels can be an important anatomical risk factor for BRVO. The evidence from this study suggests that eyes with the P1 retinal vessel position are less likely to be affected by BRVO; however, eyes with P2 and P2 variants (particularly P4) are at risk for BRVO. Furthermore, we found a causeand-effect relationship between the topographical retinal vessel positions and the number of a/v crossing points. Thus, these two factors should be considered together in risk analyses of BRVO. 


\section{Summary}

\section{What was known before}

- Arteriovenous $(\mathrm{a} / \mathrm{v})$ crossing sites are important in the pathogenesis of branch retinal vein occlusion.

- As a result of atherosclerosis, the stiffness of rigid, thickwalled arteries causes venous compression in the common adventitial sheath at the site of $\mathrm{a} / \mathrm{v}$ crossings.

- In almost all BRVO eyes, the artery is anterior to the vein at the site of an occlusion.

\section{What this study adds}

- In this pilot study, we grouped the retinal images into four topographical retinal vessel positions (P1, P2, P3, and P4) according to the outer or inner course of the main temporal retinal arteries and veins relative to the foveal center.

- We report that the topographical distribution of temporal retinal vessels might be an important anatomically predisposing risk factor for developing branch retinal vein occlusion.

- Furthermore, we found a cause-and-effect relationship between the topographical retinal vessel positions and the number of $\mathrm{a} / \mathrm{v}$ crossing points

\section{Conflict of interest}

The authors declare no conflict of interest.

\section{References}

1 Laouri M, Chen E, Looman M, Gallagher M. The burden of disease of retinal vein occlusion: review of the literature. Eye 2011; 25: 981-988.

2 Rogers SL, McIntosh RL, Lim L, Mitchell P, Cheung N, Kowalski JW et al. Natural history of branch retinal vein occlusion: an evidence-based systematic review. Ophthalmology 2010; 117: 1094-1101.

3 Hayreh SS. Prevalent misconceptions about acute retinal vascular occlusive disorders. Prog Retin Eye Res 2005; 24: 493-519.

4 Kolar P. Risk factors for central and branch retinal vein occlusion: a meta-analysis of published clinical data. J Ophthalmol 20142014; 724780.

5 Cugati S, Wang JJ, Rochtchina E, Mitchell P. Ten-year incidence of retinal vein occlusion in an older population: the Blue Mountains Eye Study. Arch Ophthalmol 2006; 124: 726-732.

6 Klein R, Klein BE, Moss SE, Meuer SM. The epidemiology of retinal vein occlusion: the Beaver Dam Eye Study. Trans Am Ophthalmol Soc 2000; 98: 133-141.

7 Wong TY, Larsen EK, Klein R, Mitchell P, Couper DJ, Klein BE et al. Cardiovascular risk factors for retinal vein occlusion and arteriolar emboli: the Atherosclerosis Risk in Communities and Cardiovascular Health studies. Ophthalmology 2005; 112: 540-547.
8 Yasuda M, Kiyohara Y, Arakawa S, Hata Y, Yonemoto K, Doi $Y$ et al. Prevalence and systemic risk factors for retinal vein occlusion in a general Japanese population: the Hisayama study. Invest Ophthalmol Vis Sci 2010; 51: 3205-3209.

9 Lim LL, Cheung N, Wang JJ, Islam FM, Mitchell P, Saw SM et al. Prevalence and risk factors of retinal vein occlusion in an Asian population. Br J Ophthalmol 2008; 92: 1316-1319.

10 Liu W, Xu L, Jonas JB. Vein occlusion in Chinese subjects. Ophthalmology 2007; 114: 1795-1796.

11 Klein R, Moss SE, Meuer SM, Klein BE. The 15-year cumulative incidence of retinal vein occlusion: the Beaver Dam Eye Study. Arch Ophthalmol 2008; 126: 513-518.

12 Ho JD, Tsai CY, Liou SW, Tsai RJ, Lin HC. Seasonal variations in the occurrence of retinal vein occlusion: a fiveyear nationwide population-based study from Taiwan. Am J Ophthalmol 2008; 145: 722-728.

13 Kawasaki R, Nagano E, Uno M, Okada M, Kawasaki Y, Kitamura A. Retinal vascular features associated with risk of branch retinal vein occlusion. Curr Eye Res 2013; 38: 989-993.

14 Jaulim A, Ahmed B, Khanam T, Chatziralli IP. Branch retinal vein occlusion: epidemiology, pathogenesis, risk factors, clinical features, diagnosis, and complications. An update of the literature. Retina 2013; 33: 901-910.

15 Staurenghi G, Lonati C, Aschero M, Orzalesi N. Arteriovenous crossing as a risk factor in branch retinal vein occlusion. Am J Ophthalmol 1994; 117: 211-213.

16 Feist RM, Ticho BH, Shapiro MJ, Farber M. Branch retinal vein occlusion and quadratic variation in arteriovenous crossings. Am J Ophthalmol 1992; 113: 664-668.

17 Rehak J, Rehak M. Branch retinal vein occlusion: pathogenesis, visual prognosis, and treatment modalities. Curr Eye Res 2008; 33: 111-131.

18 Christoffersen NL, Larsen M. Pathophysiology and hemodynamics of branch retinal vein occlusion. Ophthalmology 1999; 106: 2054-2062.

19 Jefferies P, Clemett R, Day T. An anatomical study of retinal arteriovenous crossings and their role in the pathogenesis of retinal branch vein occlusions. Aust N Z J Ophthalmol 1993; 21: 213-217.

20 Zhao J, Sastry SM, Sperduto RD, Chew EY, Remaley NA. Arteriovenous crossing patterns in branch retinal vein occlusion. Ophthalmology 1993; 100: 423-428.

21 Duker JS, Brown GC. Anterior location of the crossing artery in branch retinal vein obstruction. Arch Ophthalmol 1989; 107: 998-1000.

22 Kumagai K, Tsujikawa A, Muraoka Y, Akagi-Kurashige Y, Murakami T, Miyamoto K et al. Three-dimensional optical coherence tomography evaluation of vascular changes at arteriovenous crossings. Invest Ophthalmol Vis Sci 2014; 55: 1867-1875.

23 Muraoka Y, Tsujikawa A, Murakami T, Ogino K, Kumagai K, Miyamoto $\mathrm{K}$ et al. Morphologic and functional changes in retinal vessels associated with branch retinal vein occlusion. Ophthalmology 2013; 120: 91-99.

24 Mainster MA. The fractal properties of retinal vessels: embryological and clinical implications. Eye (Lond) 1990; 4(Pt 1): 235-241. 